\title{
Uterus didelphys with a successful term pregnancy
}

\author{
Smriti Anand*, Soniya Dhaiya
}

Department of Obstetrics and Gynecology, PGIMS, Rohtak, Haryana, India

Received: 16 February 2019

Accepted: 31 May 2019

\section{*Correspondence:}

Dr. Smriti Anand,

E-mail: smriti35@gmail.com

Copyright: ( ) the author(s), publisher and licensee Medip Academy. This is an open-access article distributed under the terms of the Creative Commons Attribution Non-Commercial License, which permits unrestricted non-commercial use, distribution, and reproduction in any medium, provided the original work is properly cited.

\begin{abstract}
Mullerian duct anomalies are congenital defects of female genital system that arise from abnormal embryological development of mullerianducts. Uterusdidelphys also known as double uterus is one of the least mulleriananomalies. Here we present a case of a woman with uterus didelphys and her successful full pregnancy.

Patient came to our hospital with diagnosis of G1P0L0 with 39 weeks with antepartum hemorrhage with active bleeding. On ultrasound she was found to be type iv placenta previa, in view of same we had done her lower segment cesarean section with delivery of a $2.5 \mathrm{~kg}$ female baby with intraoperative findings of two uterus and two cervix i.e uterus didelphys in which pregnancy was present in right uterus, placenta covering os. Post operative period was uneventful. Patient who have uterus didelphys due to mullerian defect not associated with difficulty in conception but there is increase incidence of preterm labour, malpresentation and malposition. Howeverwith appropriate antenatal and intrapartum surveillance pregnancy outcome are good.
\end{abstract}

Keywords: Anomalies, Mullerian, Uterus didelphys

\section{INTRODUCTION}

Mullerian duct anomalies are congenital anomalies of the female genital tract arising from abnormal embryological development of the mullerian ducts. These abnormalities can include failure of development, fusion, canalization, or reabsorption, which normally occurs between 6 and 22 weeks in utero. Most sources estimate an incidence of these abnormalities to be 0.5 to $5.0 \%$ in the general population. ${ }^{1-4}$ The uterus is formed during embryogenesis by the fusion of the two paramesonephric ducts (mullerian ducts). The two mullerian ducts normally fuse to form the single uterine body. Uterus Didelphys is a congenital malformation of the Mullerian duct that occurs because of complete failure of fusion of the ispilateral ducts. It results in the development of a longitudinal vaginal septum, two vagina, and two hemi-uteri each with its separate ovary and fallopian tube. The incidence of this anomaly is 1 in $3000 .{ }^{4}$ It is found to be the second least common at $8.3 \%$ of all MDAs. It remains a challenge to the obstetricians especially when it is undiagnosed before onset of labor. Patients are usually asymptomatic, but the anomaly may be associated with dysmenorrhoea, dyspareunia, infertility, spontaneous abortion, preterm labor, fetal malpresentation, intrauterine growth restriction, PROM, renal agenesis, decreased live births and caesarean delivery. ${ }^{1-6}$

Diagnosis is usually initiated by the findings of a longitudinal vaginal septum and two vaginal openings during a vaginal examination. The modalities for correct diagnosis frequently used include highly invasive methods such as hysteroscopy, hysterosalpingography, and laparoscopy/laparotomy. However, these methods rely on the clinician's subjective interpretation rather than strict diagnostic criteria. ${ }^{7}$ A 2 D ultrasound is usually the first type of imaging done; however, it is inadequate for diagnosis as it cannot reliably differentiate between subtypes of MDAs. The use of 3D ultrasound is becoming more commonly used for diagnosis as it is not only noninvasive, but it also overcomes the limitation of 
$2 \mathrm{D}$ ultrasound by providing a coronal view that enables examination of both the endometrial cavity and uterine fundus, thus giving all the information needed for morphological classification. ${ }^{3,7}$ Magnetic resonance imaging is also just as accurate and valuable in diagnosing MDAs as hysterosalpingograms, hysteroscopy, and laparoscopy are, even more so as it is noninvasive and can diagnose associated urinary tract abnormalities at the same time. ${ }^{8}$ This report discusses a case of didelphys uterus that successfully conceived, carried her pregnancy to term, and delivered by caesarean delivery without any significant complications.

\section{CASE REPORT}

A 23 years of age female admitted to our labour room with diagnosis of primigravida with 39 weeks 4 day pregnancy with antepartum hemmorhage. She presented in our department with chief complaints of bleeding per vaginum since 2 hours. On examination her pulse rate was 90/min, blood pressure was $130 / 90 \mathrm{~mm} \mathrm{Hg}$, afebrile, chest was clear. On per per Abdomen examination uterus was term size, longitudinal lie, cephalic presentation, FHS +/R, relaxed. On local examination bleeding was present.

On ultrasound, placenta was found to be posterior and covering os. In view of above we done her emergency caesarean section with delivery of a female baby of 2.5 $\mathrm{kg}$. On examination there was another uterus left to the gravid uterus with which left fallopian tube was attached and left ovary was also attached with ovarian ligament.it was found to be a case of uterus didelphys (Figure 1). Both the uterus had seprate cervices opening into seprate vaginas on per speculum examination (Figure 2). Bilateral kidneys were palpated and were normal. Fetus was delivered from right uterus. right tube and right ovary was also attached to the gravid uterus which is on right side. the uterus was closed in double layers and abdomen was closed in layers after maintaining hemostasis. postoperative period was uneventful and patient was discharged on $5^{\text {th }}$ day.

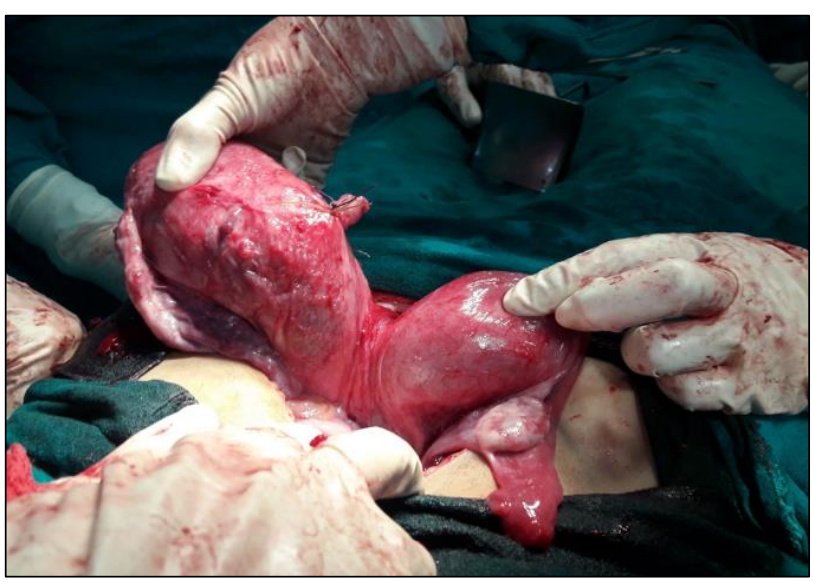

Figure 1: Two seprate uterus with gravud uterus on left side and non gravid on right side.

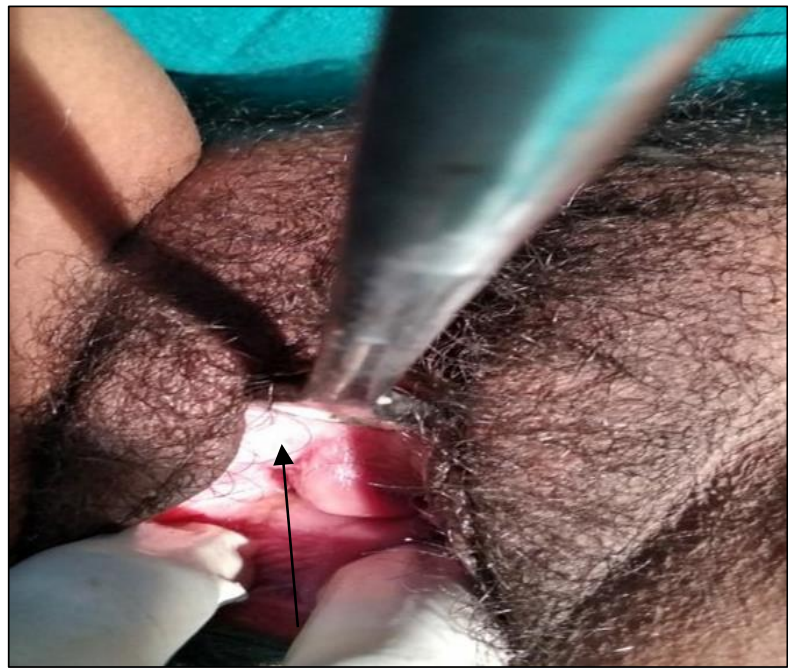

Figure 2: Arrow showing one septa between two separate vagina retracted by ant vaginal wall retracter.

\section{DISCUSSION}

Mullerian duct anomalies are congenital anatomic abnormalities that arise from non development or non fusion or failed resorption of Mullerian ducts. Mullerian anomalies prevalence is exactly unknown. But recent study shows it is 0.1 to $10 \%$. Incidence of singleton pregnancy in uterine didelphys is 1 in 3000, incidence of twin gestations is 1 in 5 million, and incidence of triplets in uterine didelphys is 1 in 25 million. ${ }^{6}$ This anomaly accounts for $11 \%$ of uterine malformations and is typically asymptomatic. ${ }^{9}$ Patients with a double uterus may need special attention during pregnancy as premature birth and malpresentation are common. Uterus didelphys in certain studies has also been found to be associated with higher rate of infertility, spontaneous abortion, intrauterine growth retardation and post-partum bleeding. ${ }^{9}$

\section{Embryology}

The first stage of mullerian duct development begins at an approximately 6 weeks gestational age when the paired mullerian ducts invaginate and then grow caudally and cross over the Wolffian ducts to meet at the midline. The subsequent three phases (fusion, resorption and vaginal induction) proceed in an orderly fashion from 9th to the $22^{\text {nd }}$ gestational week. A didelphic uterus is suggestive of an embryologic arrest occuring during the 8th week of gestation which ultimately affects the Mullerian and Metanephric ducts. However, if one of the Wolffian ducts is absent, the kidney and ureter on the ipsilateral side will fail to fuse at midline. This process may occur completely or incompletely. If the failure to fuse is complete, then a uterine didelphys is formed The most common renal tract anomaly associated with MDA is renal agenesis with right sided prevalence. ${ }^{9}$ 


\section{Classification}

Mullerian duct anomalies are classified according to the American fertility society criteria as follows:

- Class I or uterine hypoplasia or agenesis

- Class II or unicornuate uterus: A banana shaped uterus with a single fallopian tube. A rudimentary horn (communicating or non communicating) may be present.

- Class III or uterine didelphys: Two complete uteruses, each with its own cervix. A sagittal vaginal septum is seen in the majority of cases.

- Class iv or bicornuate uterus: Two uterine cavities with one cervix with an intercornual distance of $>4 \mathrm{~cm}$ and convexity fundal contour or an external fundal cleft of $>1 \mathrm{~cm}$ in depth.

- Class V or septate uterus: A fibrous septum is seen that appear hypointense on $\mathrm{T} 2 \mathrm{~W}$ images. While the muscular septum appears intermediate in intensity. MRI criteria includes a convex or flat external fundal contour or external fundal cleft of $<1 \mathrm{~cm}$ in depth.

- Class VI or arcuate uterus: It is a normal variant and is characterized by convex contour of fundus with fundal endometrial indentation.

- Class VII or diethylbestrol induced: exposure to this synthetic estrogen antenatally can result in a $\mathrm{T}$ shaped, hypoplastic and constricted uterus. Our case matched with class iii Mullerian ducts anomaly.

Most women with a didelphys uterus are asymptomatic, but may present with dyspareunia or dysmenorrhea in the presence of a thick, sometimes obstructing, vaginal septum. This obstructing vaginal septum can lead to hematocolpos/hematometrocolpos and thus present as chronic abdominal pain as well. Rarely, genital neoplasms and endometriosis are reported in association with cases of didelphys uterus. ${ }^{9}$

Frank breech is most frequent abnormal presentation in uterine didelphys. Management in patients with a double uterus may need special attention during pregnancy, as premature birth and malpresentations are common. Other complications of pregnancy are cervical incompetence, PPH, uterine rupture. As far as mode of delivery in patients with uterus didelphys, opinion is divided into normal vaginal delivery or elective cesarean section. Vaginal delivery has been accomplished merely by excision of vaginal septum.

\section{Diagnosis}

A pelvic examination will typically reveal a double vagina and a double cervix. Investigations are usually prompted on the basis of such findings as well as when reproductive problems are encountered. Helpful techniques to investigate the uterine structure are.
- Transvaginal

ultrasonography

and

$$
\text { sonohysterography }
$$

- Hysterosalpingography

- MRI and hysteroscopy

- Laparoscopy/laparotomy.

Because of high incidence of abnormal presentations and cervical incompetence, most of the people prefer cesarean section.

Further, because the Mullerian ducts develop often in association with Wolffian ducts, abnormalities of the kidneys may be found in conjunction with uterine abnormalities.

More recently 3-D ultrasonography has been advocated as an excellent non-invasive method to evaluate uterine malformations, it accurately analyses uterine structure, contour of fundus, muscular thickness and septum length. It is best performed during secretory phase of menstrual cycle.

Aci'en's prospective observational $s^{2}{ }^{2}$ of the reproductive outcome of women with different uterine anomalies in comparison to a normal uterus found the rate of term delivery for a didelphys uterus significantly lower than the normal uterus group but the rate was not as low as that of the bicornuate group and septate group5.On the other hand, a large retrospective longitudinal study of 3181 patients by Raga et al. demonstrated poor reproductive performance in women with didelphys uteri with a higher rate of preterm delivery, spontaneous abortion, and the lowest chance of having a term delivery than the other MDAs. ${ }^{3}$ In addition, a long term retrospective follow-up of 49 women with didelphys uterus found no impairment with fertility and decreased rate of spontaneous abortion; however the rate of prematuritywas increased in comparison to other known studies on septate and bicornuate uteri. ${ }^{2}$

A didelphys uterus is not an indication for cesarean delivery and thus vaginal delivery should be considered first. Finally, cervical incompetence is not usually associated with didelphys uterus and thus cerclage is not routinely used unless there is a history of cervical incompetence or premature dilation is found on examination during early second trimester. ${ }^{2}$ A didelphys uterus has been shown in many case reports to occur as a part of a syndrome, more specifically called, HerlynWerner-Wunderlich (HWW) syndrome, also known as obstructed hemivagina and ipsilateral renal anomaly(OHVIRA). It is a very rare congenital anomaly of the urogenital tract involving Mullerian ducts and Wolffian structures, and it is characterized by the triad of didelphys uterus, obstructed hemivagina, and ipsilateral renal agenesis. ${ }^{9}$ This condition can cause hematometrocolpos or hematocolpos on the side of the obstructed hemivagina which produces a mass effect with subsequent lower abdominal pain. ${ }^{8}$ Most cases present 
after menarche as intense lower abdominal pain and/or a protruding mass over the vaginal introitus. ${ }^{8}$

\section{CONCLUSION}

Mullerian duct anomalies are rare and usually associated with various clinical manifestations which range from dysmenorrhoea at puberty to preterm delivery and increased rate of caesarean section during reproductive age group. So through investigations should be done in cases of spasmodic dysmenorrhoea and case of second trimester recurrent abortions. Overall the literature available on the didelphys uterus is quite limited at the present time. Therefore more studies are needed in order to better determine the reproductive and gestational outcomes, so that clinicians can adequately advise and care for their patients. Clinicians should have high index of suspicion of uterine anomaly to make early diagnosis of uterus Didelphys. Pregnancy in a uterus Didelphys deserves early diagnosis of the anomaly, and meticulous care in pregnancy and delivery to avert the associated adverse outcomes.

Funding: No funding sources Conflict of interest: None declared

Ethical approval: Not required

\section{REFERENCES}

1. Heinonen PK. Uterus didelphys: a report of 26 cases. Eur J Obstet Gynecol Reprod Biol. 1984;17(5):34550.

2. Acién P. Reproductive performance of women with uterine malformations. Hum Reprod. 1993;8(1):1226.
3. Raga F, Bauset C, Remohi J, Bonilla-Musoles F, Simon C, Pellicer A. Reproductive impact of congenital Mullerian anomalies. Hum Reprod. 1997;12(10):2277-81.

4. Heinonen PK. Clinical implications of the didelphic uterus: long-term follow-up of 49 cases. Eur J Obstet Gynecol Reprod Biol. 2000;91(2):183-90.

5. Grimbizis GF, Camus M, Tarlatzis BC, Bontis JN, Devroey P. Clinical implications of uterine malformations and hysteroscopic treatment results. Hum Reprod Update. 2001;7(2):161-74.

6. Takagi H, Matsunami K, Noda K, Furui T, Imai A. Magnetic resonance imaging in the evaluating of double uterus and associated urinary tract anomalies: a report of five cases. J Obstet Gynaecol. 2003;23(5):525-7.

7. Madureira AJ, Mariz CM, Bernardes JC, Ramos IM. Case 94: Uterus Didelphys with Obstructing Hemivaginal Septum and Ipsilateral Renal Agenesis. Radiology. 2006;239(2):602-6.

8. Brown MA. MR imaging of benign uterine disease. Magn Reson Imaging Clin N Am. 2006;14(4):43953.

9. Zhang Y, Zhao YY, Jie Q. Obstetric outcome of women with uterine anomalies in China. Chin Med J. 2010;123(4):418-22.

Cite this article as: Anand S, Dhaiya S. Uterus didelphys with a successful term pregnancy. Int J Reprod Contracept Obstet Gynecol 2019;8:2906-9. 